\title{
Prenatal-screening companies expand scope of DNA tests
}

\section{But the increasingly accurate analyses carry the ethical dilemma of uncertain outcomes.}

\section{BY ERIKA CHECK HAYDEN}

W hen the small piece of chromosome 22 associated with DiGeorge syndrome is missing, this can cause developmental abnormalities such as speech and language delays, cleft palate, a faulty immune system and heart defects. Or an apparently healthy baby can be born.

With non-invasive genetic screening on the rise, companies are racing to add chromosomal abnormalities such as that associated with DiGeorge syndrome to the list of disorders for which they can test. But because the effects of these genetic anomalies are sometimes uncertain, the test results may prompt difficult decisions for families who receive such diagnoses.

The tests are a welcome development, says Philippa Brice, communications director of the non-profit PHG Foundation in Cambridge, UK, which promotes the responsible use of biomedical science. But she adds, "there is a risk that women may receive early in pregnancy a result they do not fully understand".

The issue is becoming more common as genetic-screening companies demonstrate the accuracy of the tests - and press their case that all pregnant women should be screened. On 26 February, scientists at Illumina, a genomesequencing company headquartered in San Diego, California, announced the results of a study comparing its genetic test, called verifi, with the standard biochemical screening routinely offered to pregnant women (D. W. Bianchi et al. N. Engl. J. Med. http://doi. org/rqn; 2014). The genetic test checks directly for abnormalities in fetal DNA that crosses the placenta and enters the mother's bloodstream, whereas the biochemical screen estimates the risk of chromosomal disorders indirectly, by measuring proteins and hormones in the mother's blood that are linked with higher rates of birth defects. If either screen suggests the presence of a defect, it is usually confirmed by an invasive test, such as an amniocentesis.

The study, which was funded by Illumina, screened more than 1,900 US women for chromosomal abnormalities, including Down's syndrome, using both the biochemical and genetic methods. The verifi test had a false-positive rate of $0.3 \%$, about one-tenth of that associated with biochemical screening. The finding is crucial because most physicians' groups recommend that screening be used only in women at

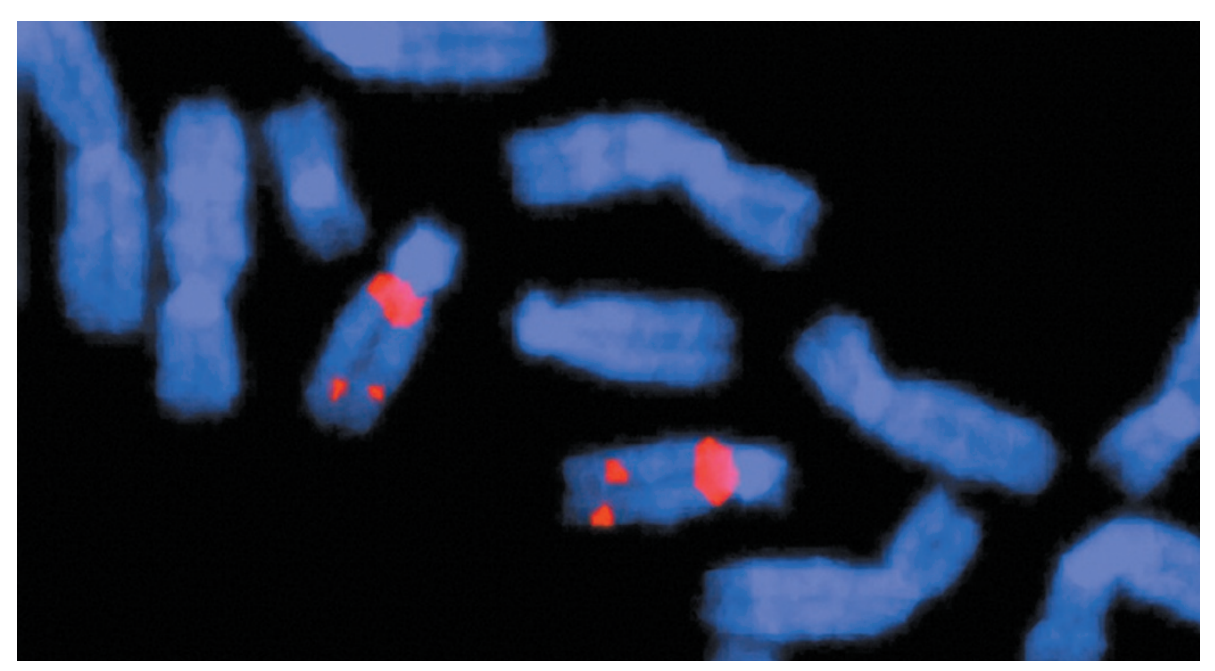

DNA deletions in chromosome 22 (indicated by pink markers) are associated with DiGeorge syndrome.

high risk of carrying a fetus with chromosomal disorders - mainly women aged over 35 - to minimize the chance that a false positive would lead to an invasive test. The study examined women with an average age of just under 30 .

With such a low false-positive rate, the genetic screening should be offered to women of all ages, says Anthony Gregg, a geneticist and obstetrician at the University of Florida in Gainesville who was the lead author of geneticscreening guidelines published last April by the American College of Medical Genetics and Genomics in Bethesda, Maryland.

Increased acceptance could expand the already growing market for genetic screen-

\section{"The companies are all trying} to one-up each other." ing. Sequenom, a sequencing company based in San Diego, said in January that it performed 148,500 of its MaterniT21 tests in 2013, up 140\% from 2012. And in November, Ariosa of San Jose, California, said that it had performed its Harmony test on 150,000 women since launching in May 2012. Most tests are performed in the United States, although the companies are preparing to roll them out globally.

Companies are trying to stand out by expanding the number of conditions their tests check for. Microdeletions and microduplications - genetic defects that can be millions of base pairs long - are seen as the first step, although some companies have designs to sequence the entire fetal genome. "The companies are all trying to one-up each other," says Wendy Chung, a paediatrician and geneticist at Columbia University in New York City. "It's not clear where the number will stop."

Typically, genetic and biochemical screening tests have scanned only for missing or extra copies of whole chromosomes, which are known to result in clear outcomes. An extra copy of chromosome 21, for instance, causes Down's syndrome. But last October, Sequenom began looking for microdeletions within chromosomes with its MaterniT21PLUS test. Natera, based in San Carlos, California, added tests for microdeletions to its Panorama test last month. And Illumina says that it will follow suit and expand the capabilities of its verifi test in the next few months. "Nobody in the twentyfirst century should have a pregnancy without being screened for these microdeletions," says Matthew Rabinowitz, Natera's chief executive.

Routine checking for microdeletions could enable more families to prepare for children with special needs. But because such microdeletions are smaller than whole-chromosome abnormalities, they affect fewer genes, and the physical or mental effects are not always predictable. Chung, for instance, knows of many adults who found out that they had DiGeorge syndrome only after their children were diagnosed and they decided to get tested themselves. She says that as tests expand their scope and offer less-certain predictions, more families will face tough choices about their unborn children. 